\title{
The Effects of Amplification and Dilution Agent in Transmitting Sin Nombre Virus (SNV) in Deer Mouse Population
}

\author{
${ }^{1}$ L. W. F. Lee*, and ${ }^{1,2}$ M. H. Mohd \\ ${ }^{1}$ School of Mathematical Sciences \\ Universiti Sains Malaysia (USM) \\ 11800 USM, Penang, Malaysia \\ ${ }^{2}$ School of Mathematical Sciences, Faculty of Science and Technology \\ Universiti Kebangsaan Malaysia (UKM) \\ 43600 Bangi, Selangor, Malaysia \\ *Corresponding author: lloydlwf1993@gmail.com
}

Article history

Received: 1 October 2019

Received in revised form: 21 April 2020

Accepted: 8 July 2020

Published online: 1 August 2020

\begin{abstract}
Numerous studies have linked biodiversity with zoonotic disease control. However, researchers have warned against simply believing that the increase in biodiversity can reduce infectious disease in the community (i.e. the dilution effect). They proposed that the amplification effect (increase in biodiversity accompanied by an increase in disease prevalence) might sometimes occur. In this study, we formulated a deterministic model to consider the impact of an amplification or dilution agent on the SNV transmission in the deer mouse population. Bifurcation analysis was carried out to examine the combined influences of the environmental carrying capacity, the interspecific competition strength and the impact of amplification or dilution agent on the deer mouse population. Our results showed that the system with amplification agent required a higher carrying capacity or stronger interspecific strength to compensate for its amplification effect in suppressing the SNV prevalence. This observation may explain some of the empirical observations which noted the lack of reduction in SNV prevalence despite the high species diversity observed at those sites. To conclude, we highlight the importance of investigating the roles played by the other species in an assemblage to better understand their relationship with the SNV prevalence in deer mouse population.
\end{abstract}

Keywords Sin Nombre Virus (SNV); Biodiversity; Amplification effect; Dilution effect; Bifurcation.

Mathematics Subject Classification 97M10.

\section{Introduction}

The importance of biodiversity has been a significant issue in disease ecology over the years. It is believed that biodiversity effect can determine the spread of infectious disease in the 
ecosystem, for example, the vector-borne Lyme disease [1]. However, the mechanism behind this principle is rather complex and may differ between the type of diseases. The effect of biodiversity on the zoonotic ecological system can be generalized into either the "dilution effect" or the "amplification effect". The dilution effect is said to occur when the presence of additional species or an increase in species richness reduces the prevalence of the disease; the amplification effect is observed when the increase in diseases prevalence is accompanied by an increase in diversity [2]. Hence, Randolph and Dobson [3] warned against jumping on the bandwagon of biodiversity protects against disease without further investigation on the mechanism behind it. They highlighted the occurrence of dilution or amplification effect is likely dependent on the specific community composition instead (refer to [4] for a case study example on hantavirus). Interestingly, the occurrence of both dilution and amplification effects may occur concurrently, as observed in [5], in the deer mouse population, which is a host for the Sin Nombre virus (SNV). The authors managed to observe a reduction in the deer mouse density (dilution effect) as well as an increase in transmission rate of the hantavirus within the deer mouse population (amplification effect) when small mammal diversity increases. Their study concluded with the occurrence of a net dilution for the SNV prevalence as the dilution effect was the larger of the two effects. To better understand the dynamics of such occurrence in the deer mouse population, we formulated a deterministic mathematical model based on the work of [6] and [5] and postulated that the carrying capacity of the assemblage and influences of the interspecific competition strength could have effects on the multi-species community compositions.

Before the model is presented, a brief introduction about hantavirus disease, particularly on SNV, would be given to understand the traits of the virus and the reasons why the proposed model was constructed as such. Hantavirus is a genus virus of the Bunyaviridae family. Humans, whom had contact with the saliva, urine and excreta of the hantavirus infected animals, may develop hemorrhagic fever with renal syndrome (HFRS) or hantavirus pulmonary/cardiopulmonary syndrome (HPS/HCPS) depending on the virus strain [7]. HPS has around $40 \%$ mortality rate in humans, and SNV, which is primarily hosted by deer mouse, Peromyscus maniculatus, is one of the virus strains that caused HPS [8]. Following the HPS outbreak in the Southwest United States in 1993, numerous studies, both theoretical and empirical, have been carried out to better examine the relationship between the prevalence of SNV and the deer mouse population [9-11]. SNV is a lifelong infection in the deer mouse [12] and is transmitted horizontally through aggressive encounters between the rodents [13].

Following the footstep of the observed dilution effect on the Lyme disease, several observational and experimental studies have been carried out to investigate the impact of biodiversity on the non-vector borne SNV prevalence in the deer mouse population [5, 14-17]. While these studies managed to observe the dilution effect, the mechanisms that affect the spread of this disease differ between them. Some observed a reduction in host density while others observed a decrease in encounter rate (which directly influences the disease transmission rate) following the increment of species diversity. As such, we hope our proposed mathematical model would provide further insights into these contrasting observations.

\section{Proposed Model}

For simplicity purpose, the proposed model would describe the effects of a non-host species onto the SNV prevalence in the deer mouse population (a non-host species is defined as a species 
other than the deer mouse which would not be infected by the SNV). This model was inspired by Peixoto and Abramson's "single host, single non-host" (abbreviated as PA model) approach [6] and Luis et al. density-dependent restricted population growth SI model (abbreviated as Luis model) [5]. Before the proposed model is introduced, the Luis and PA models would be briefly discussed to give the readers an idea on what they described.

The Luis model was formulated to study the dynamics of the long-term SNV persistence among the deer mouse population [18]. In this endemic model, there is an immigration of SNV infected deer mouse into the system, and the SNV can induce death among the deer mouse. The following depicts the mathematical descriptions of the Luis model.

$$
\begin{aligned}
& \frac{d S}{d t}=N\left(b-a r \frac{N}{K}\right)-S\left(d+(1-a) r \frac{N}{K}\right)-\beta S I \\
& \frac{d I}{d t}=\beta S I-I\left(\mu+d+(1-a) r \frac{N}{K}\right)+\phi
\end{aligned}
$$

where $S$ is the susceptible deer mouse, $I$ is the infected deer mouse, $N$ is the total deer mouse population $(N=S+I)$. The descriptions for the rest of the parameter can be found in Table 1 .

Table 1: Descriptions of the Parameters in the System (1)

\begin{tabular}{|c|l|}
\hline Parameters & \multicolumn{1}{|c|}{ Descriptions } \\
\hline$b$ & Birth rate of deer mouse \\
\hline$d$ & Death rate of deer mouse \\
\hline$r$ & $b-d$ \\
\hline$a$ & $\begin{array}{l}\text { The proportion of density dependence due to density dependence } \\
\text { in birth rates }\end{array}$ \\
\hline$\mu$ & Disease-induced mortality rate \\
\hline$K$ & Carrying capacity \\
\hline$\beta$ & SNV transmission rate \\
\hline$\phi$ & Immigration rate of $I$ \\
\hline
\end{tabular}

Note: Readers can refer to [5] for more details of the model and parameters

To account for the biodiversity effect, the PA model describes the SNV prevalence in the presence of a non-host species. Unlike the Luis model, the PA model studies the rapid shortterm outbreaks(epidemic state) of SNV prevalence in the presence of a non-host species. The PA model was constructed by combining the Lotka-Volterra two species competition model with Abramson and Kenkre model [11], which describes the epidemic state of the SNV prevalence 
in the deer mouse population. The PA model is as follows.

$$
\begin{aligned}
& \frac{d S}{d t}=b_{1}(S+I)-d_{1} S-\frac{S\left(S+I+q_{1} Z\right)}{K_{1}}-a S I \\
& \frac{d I}{d t}=-d_{1} I-\frac{I\left(S+I+q_{1} Z\right)}{K_{1}}+a S I \\
& \frac{d Z}{d t}=b_{2} Z-d_{2} Z-\frac{Z\left[Z+q_{2}(S+I)\right]}{K_{2}}
\end{aligned}
$$

where $S, I$ and $Z$ represent the susceptible deer mouse, infected deer mouse and non-host population. The description of the other parameters is presented in Table 2.

Table 2: Descriptions of the Parameters in the System (2)

\begin{tabular}{|c|l|}
\hline Parameters & \multicolumn{1}{c|}{ Descriptions } \\
\hline$b_{1}$ & The birth rate of deer mouse population \\
\hline$d_{1}$ & The death rate of deer mouse population \\
\hline$K_{1}$ & The carrying capacity of the deer mouse population \\
\hline$a$ & The SNV transmission rate among deer mouse population \\
\hline$b_{2}$ & The birth rate of the non-host population \\
\hline$d_{2}$ & The death rate of the non-host population \\
\hline$K_{2}$ & The carrying capacity of the non-host population \\
\hline$q_{1}$ & $\begin{array}{l}\text { The interspecific competition strength exerted by the non-host } \\
\text { population onto the deer mouse population }\end{array}$ \\
\hline$q_{2}$ & $\begin{array}{l}\text { The interspecific competition strength exerted by the deer mouse } \\
\text { population onto the non-host population }\end{array}$ \\
\hline
\end{tabular}

To construct the proposed model, a non-host species is introduced into the Luis model. This idea is similar to how PA model was formulated.Unlike the PA model, the proposed model allows the non-host to have a direct influence on the SNV transmission rate. This effect was incorporated to account for the observed transmission rate increment along with the increasing small mammal diversity (see [5]). We believed it was a sensible choice as the additional species may act as the dilution or amplification agent [2].

The proposed model is as such

$$
\begin{aligned}
\frac{d m_{\mathrm{s}}}{\mathrm{d} t} & =N\left[b_{1}-a r_{1}\left(\frac{N+q_{1} z}{K_{1}}\right)\right]-m_{\mathrm{s}}\left[d_{1}+r_{1}(1-a)\left(\frac{N+q_{1} z}{K_{1}}\right)\right]-\gamma(1+\delta z) m_{\mathrm{s}} m_{\mathrm{i}} \\
\frac{d m_{i}}{d t} & =\gamma(1+\delta z) m_{\mathrm{s}} m_{\mathrm{i}}-m_{\mathrm{i}}\left[\mu+d_{1}+r_{1}(1-a)\left(\frac{N+q_{1} z}{K_{1}}\right)\right] \\
\frac{d z}{d t} & =r_{2}\left[1-\frac{z+q_{2} N}{K_{2}}\right] z
\end{aligned}
$$


where $N=m_{\mathrm{s}}+m_{\mathrm{i}}$ is the total density of the host individuals, $m_{\mathrm{s}}$ is the density of the susceptible individuals, $m_{\mathrm{i}}$ is the density of the infected individuals and $z$ is the density of the non-host individuals. The descriptions for the rest of the parameters are presented in Table 3 .

Based on our literature findings, we observed that the presence of the non-host could influence the disease transmission rate. However, we were uncertain whether the influence acted on the encounter rate of the host or on the probability of transmission. Clay et al. [19] observed a decrease in contact rates along with an increase of species diversity, while Rubio et al. [20] did not find any change in the encounter rate in their experimental study. Luis et al. [5] hypothesized the change of the disease transmission rate might be due to the probability of transmission or immunity of the host, as Brenner and Moynihan [21] observed that stress could induce a decrease in the immunity of the host. Thus, we chose to express the transmission rate with $\gamma(1+\delta z)$ where we believed the density of the non-host affects the host transmission rate with a proportional constant $\delta$.

We shall present 2 case studies for this model. The first case study considered the nonhost has a relatively weaker interspecific strength than the host species and does not influence the transmission rate, e.g. the desert pocket mouse, Chaetodipuspenicillatus. The second case was to consider the non-host has a relatively stronger interspecific strength compared to the host and has a direct effect on the transmission rate, e.g. the Merriam's kangaroo rat, Dipodomysmerriami [20]. The latter case's belief was based on Rubio et al.'s findings on the avoidance behaviour of the deer mouse from the Merriam's kangaroo rat and the hypothesized stress increment due to the presence of a larger body Merriam's kangaroo rat which may indirectly influence the transmission rate. For abbreviation purposes, we shall term the nonhost in case study 1 as a dilution agent and as an amplification agent in case study 2 for the rest of this paper.

\subsection{Choice of Parameter Value}

In order to make sense of the parameter value in ecological point of view, we shall limit the values of $b_{1}, b_{2}, d_{1}, d_{2}, a, K_{1}, K_{2}, \gamma \mu>0,0<q_{1}, q_{2}<1$ (to ensure the existence of both species, see [23] Table 1) and $\delta \geq 0$ in this study. For the choice of the parameter values chosen for both studies, please refer to Table 3 .

\section{$3 \quad$ Results}

By setting the left-hand side of the equations in the system (3) to zero, we would then obtain the critical points. Due to the difficulty to solve the system analytically, we relied on the mathematical software, Maple 2016, to obtain the solutions. We obtained eight critical points $\left(m_{\mathrm{s}}{ }^{*}, m_{\mathrm{i}}{ }^{*}, z^{*}\right)$ in total.Out of the eight points, there were three negative solutions, one trivial solution, two solutions of either species goes extinct, one solution where the susceptible deer mouse coexists with the non-host, and a solution where the susceptible, infected and non-host coexisted. By solving system (3) with $K_{1}=20$ and $K_{2}=15$, the population density versus time plots are as shown in Figure 1.

Although both cases have the same carrying capacity, we noted that the population density of the infected is much higher in the case of the amplification agent (Figure 1(b)) compared to the case of the dilution agent (Figure 1(a)). This is indeed what we have expected as we 
Table 3: Descriptions and the Values of the Parameters in the System (3)

\begin{tabular}{|c|c|c|}
\hline \multirow{2}{*}{ Parameters } & \multirow{2}{*}{ Descriptions } & Parameter Value \\
\hline & & \begin{tabular}{l|l} 
Case Study 1 & Case Study 2 \\
\end{tabular} \\
\hline$K_{1}$ & The carrying capacity of the host. & 20 \\
\hline$K_{2}$ & The carrying capacity of the non-host. & 15 \\
\hline$r_{1}$ & $\begin{array}{l}\text { The net density dependent growth rate } \\
\text { for the host, } b_{1}-d_{1} \text {. }\end{array}$ & $3.1496 \times 10^{-1}$ \\
\hline$b_{1}$ & $\begin{array}{l}\text { The density dependent birth rate for } \\
\text { the host. }\end{array}$ & $0.315^{\mathrm{a}}$ \\
\hline$\mu$ & The disease induced mortality rate. & $0.085^{\mathrm{a}}$ \\
\hline$d_{1}$ & The death rate of the host. & $3.66 \times 10^{-5 a}$ \\
\hline$q_{1}$ & $\begin{array}{l}\text { The interspecific pressure exerted by } \\
\text { the non-host onto the host. }\end{array}$ & $0.2^{\mathrm{b}}$ \\
\hline$a$ & $\begin{array}{l}\text { The proportion of density dependence } \\
\text { due to density dependence of the host } \\
\text { in birth rates. }\end{array}$ & $0.614^{\mathrm{a}}$ \\
\hline$r_{2}$ & $\begin{array}{l}\text { The net density dependent growth rate } \\
\text { for the non-host, } b_{2}-d_{2} \text {. }\end{array}$ & $3.9996 \times 10^{-1}$ \\
\hline$b_{2}$ & $\begin{array}{l}\text { The density dependent birth rate for } \\
\text { the non-host. }\end{array}$ & $0.4^{\mathrm{b}}$ \\
\hline$d_{2}$ & The death rate of the non-host. & $4.0 \times 10^{-5 b}$ \\
\hline$q_{2}$ & $\begin{array}{l}\text { The interspecific pressure exerted by } \\
\text { the host onto the non-host. }\end{array}$ & $0.3^{\mathrm{b}}$ \\
\hline$\gamma$ & $\begin{array}{l}\text { Initial disease transmission rate of the } \\
\text { host without the influence of additional } \\
\text { species. }\end{array}$ & $0.0130^{c}$ \\
\hline$\delta$ & $\begin{array}{l}\text { Proportional constant of the disease } \\
\text { transmission rate with the non-host } \\
\text { density. }\end{array}$ & $0.0543^{\mathrm{d}}$ \\
\hline
\end{tabular}

${ }^{a}$ The values were based on [22].

${ }^{\mathrm{b}}$ The values for $q_{1}, b_{2}, d_{2}$ and $q_{2}$ were assumed due to the lack of data. However, we chose values that were closed to the deer mouse by assuming the non-host is of a similar rodent species.

${ }^{\mathrm{c}} \gamma$ was estimated by regressing the Simpson's D diversity index, $D=\left(\sum_{i=1}^{R} p_{i}^{2}\right)^{-1}$, where $p_{i}$ is the proportion abundance of species $i$, and $R$ is the small mammal species richness against the transmission rate, $\beta$ in [5] Table 1, with suppressed constant. Thus, $\hat{\gamma}=0.0130$ by setting $D=1.0$, where $D=1.0$ indicates the presence of the host species only/absence of species richness (refer to Table 4 for the detailed regression result).

${ }^{\mathrm{d}}$ To estimate $\delta$ for case study 2, we assumed the community consisted of 2 species only with deer mouse density being more abundance than the Merriam's kangaroo rat by a 3:2 ratio (estimated from the capture rate of the empirical study in [14]) and the Merriam's kangaroo rat density has a round up value of $z=17$, which was estimated from the deer mouse density of 26 (rounded up value of 25.65) in Cascade.11, Montana in [5]. By solving $D=\left(0.6^{2}+0.4^{2}\right)^{-1}=1+\delta z$, we obtained $\hat{\delta}=0.0543$. 
Table 4: Regression Result of Simpson's D Diversity Index against Transmission Rate.

\begin{tabular}{|c|c|c|c|c|c|}
\hline $\begin{array}{c}\text { Transmission } \\
\text { Rate }\end{array}$ & Coefficient & $\begin{array}{c}\text { Standard } \\
\text { Error }\end{array}$ & $\boldsymbol{t}$ statistics & $\boldsymbol{p}$-value & $\begin{array}{c}\text { 95\% Confidence } \\
\text { Interval }\end{array}$ \\
\hline Simpson's D & 0.0130 & 0.0015 & 8.43 & $<0.001$ & $(0.0097,0.0162)$ \\
\hline
\end{tabular}

aThe regression was carried out with the 18 observational data from [5] Table 1 with the constant variable being suppressed.

hypothesized the inclusion of any non-host into the deer mouse dynamical system will not always cause a dilution. It should also be noted that a non-host with a higher "strength" of interspecific competition with the host population may not always be effective in suppressing the prevalence of infection without further exploring its impact on the ecological behaviour of the host population, and at times, a non-host with a relatively "lower" interspecific competition strength may serve as a better diluting agent in suppressing the infection.

To further explore the dynamical system (3), bifurcation analysis up to co-dimension two was carried out primarily on $K_{1}, K_{2}$ and $q_{1}$ for both case studies. The bifurcation analyses were all done through XPPAUT's AUTO, and the co-dimension one bifurcation plots can be found in Figure 2, while Figure 3 shows the co-dimension two bifurcation diagrams.

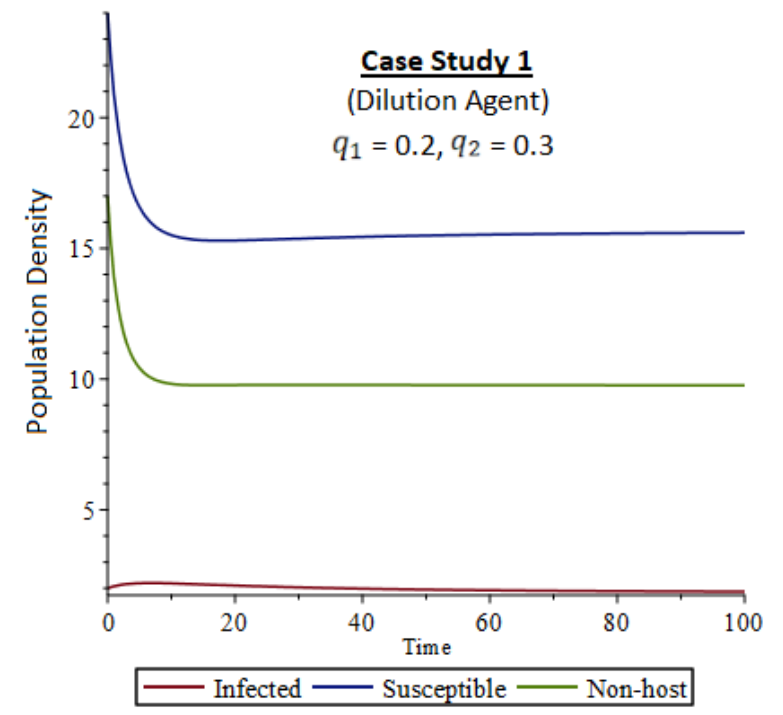

(a)

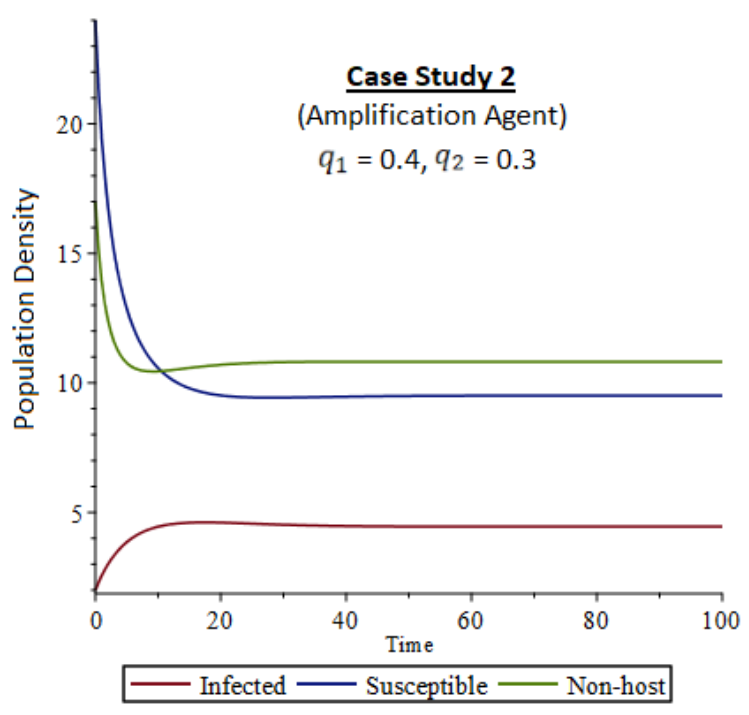

(b)

Figure 1: Population Density Versus Time Graph for Case Study 1 (a) and 2 (b) with $K_{1}=$ 20 and $K_{2}=15$, where the Infected Population Stabilised at 1.85 in Case Study 1 and 4.45 in Case Study 2

\subsection{Co-dimension 1 Bifurcation Analysis}

In Figure 2(a), there were two steady states for both cases. The horizontal line at $m_{i}=0$ represents the stead ystate where the infected population is absent while the other represents 
the growing presence of the infected population steady state. Transcritical bifurcation was observed at the intersection between these two lines as the stable steady state at $m_{i}=0$ was switched to the growing presence of the infected population stable steady state. This indicates that a certain threshold of the host's carrying capacity needs to be attained for the infection population to exist in the system. In case study 1 (dilution agent), the threshold $K_{1, \mathrm{D}}$ was larger compared to case study 2's $K_{1, \mathrm{~A}}$ (amplification agent). This indicates that it is easier for the infected population to exist in the presence of the amplification agent although its interspecific competition strength is stronger than the dilution agent (amplification agent's $q_{1}=0.4>0.2$ ) . When $K_{1}$ has reached $>60$, the stable steady state of the growing infected population for both case studies converged and resulted in similar infected population for the value of $K_{1}$ beyond that. Hence, we may conclude that the role of the non-host maybe irrelevant on the infected population when the carrying capacity of the host is enormous given that the other parameters were kept at the values presented in Table 3.

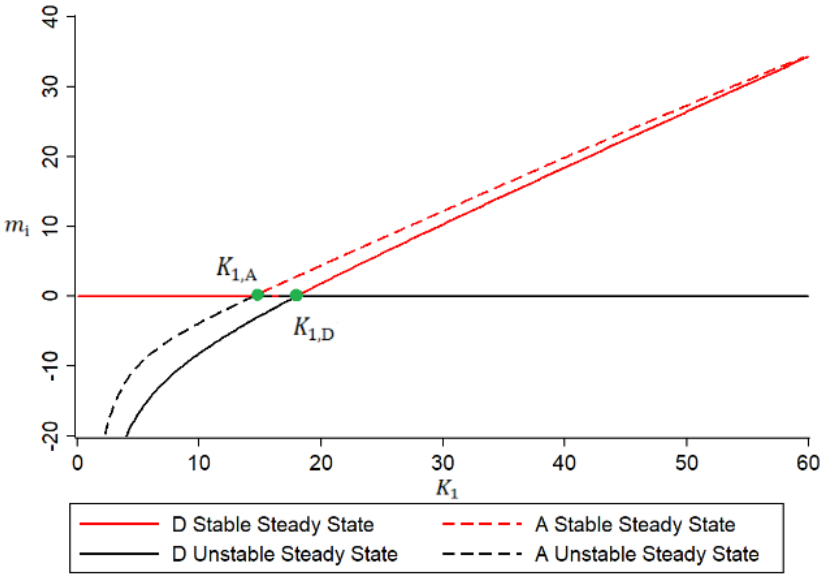

(a)

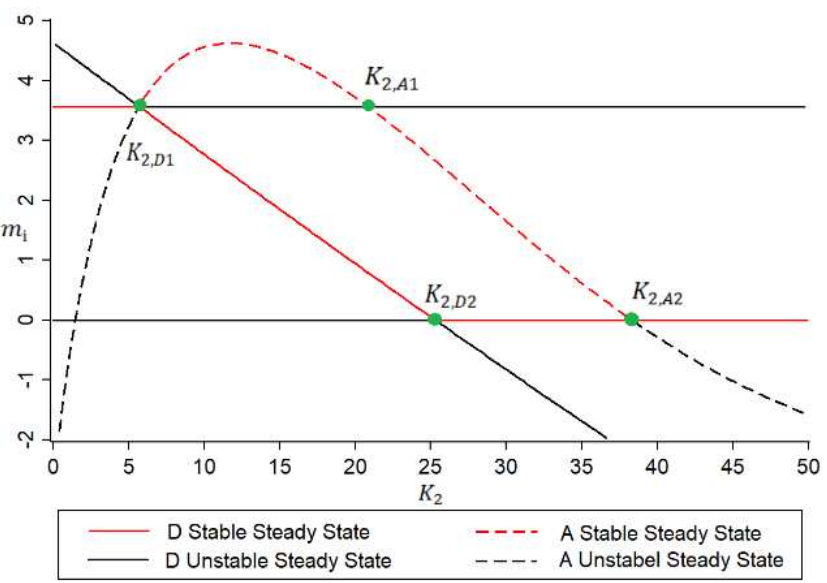

(b)

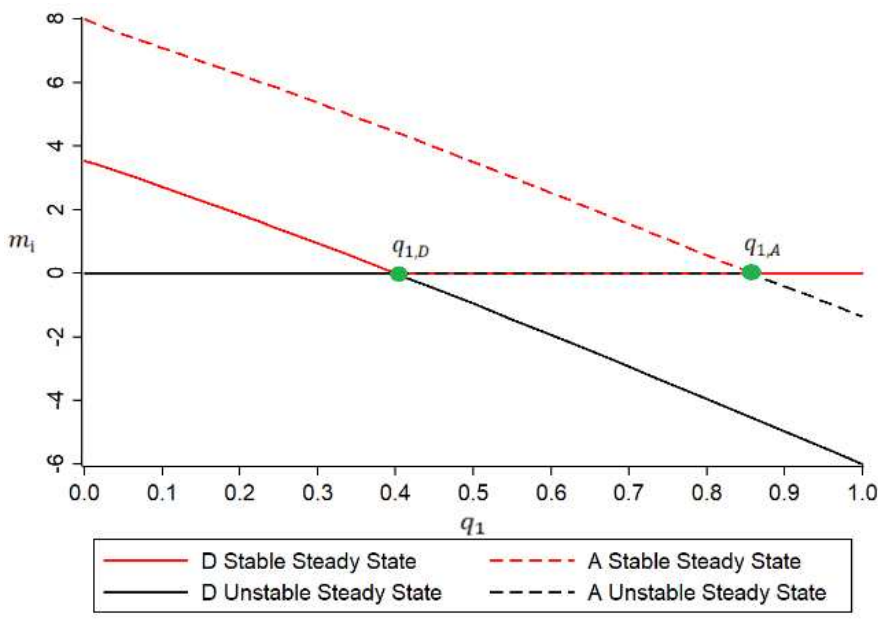

(c)

Figure 2: Co-dimension 1 Bifurcation Diagrams of $K_{1}(\mathrm{a}), K_{2}(\mathrm{~b})$ and $q_{1}(\mathrm{c})$ for the Infected Population, $m_{\mathrm{i}}$, where "D" Refers to Dilution Agent (Case Study 1) and "A" Refers to Amplification Agent (Case Study 2) 
For the bifurcation analysis on $K_{2}$ (Figure 2(b)), there were 3 steady states and 2 transcritical bifurcation points in the system. Based on Figure 2(b), case study 1 and 2 shared the same first transcritical bifurcation point, $K_{2, D 1}$. This indicated the effect of a dilution agent and an amplification agent is the same in affecting SNV prevalence if the carrying capacity of both dilution and amplification agents are below the critical threshold $K_{2, D 1}$. However, the infected population density behaved differently between case study 1 and 2 when $K_{2}>K_{2, D 1}$. For case study 1 , the infected population started to decrease after $K_{2}>K_{2, D 1}$ and eventually went extinct at the second transcritical bifurcation point, $K_{2, D 2}$. On the other hand, the infected population momentarily increased in the range of $K_{2, D 1}<K_{2}<K_{2, A 1}$ and then decreased towards the extinction point at $K_{2, A 2}$ for case study 2 . We hypothesized that this observed momentary increase represents the amplification effect of the amplifying agent. When the carrying capacity of the amplifying agent is not relatively large enough $\left(K_{2}<K_{2, A 1}\right)$, the infected population might not decrease. In the worst case, the presence of the amplifying agent will in turn promote the increment of the infected population when $K_{2, D 1}<K_{2}<K_{2, A 1}$. This phenomenon is rather counterintuitive to the idea of biodiversity protects against disease transmission. Hence, this provides support to our hypothesis that the role played by other species in the species assemblage is crucial in subduing the infection prevalence.

In Figure 2(c), both case studies had 2 steady states and only experienced 1 transcritical bifurcation. The infected deer mouse population for both cases started to decrease across $q_{1}$ and eventually went extinct after the transcritical bifurcation point, $q_{1, D}$ for case study 1 and $q_{1, A}$ for case study 2 respectively. However, the interspecific competition strength required by the amplification agent for driving out the infection was around twice the strength required by the dilution agent; thus, highlighting the efficiency of suppressing the infection lies within the role played by the other species.

\subsection{Co-dimension 2 Bifurcation Analysis}

By extending the analysis from the transcritical bifurcation points in Figure 2, we were able to obtain the co-dimension 2 bifurcation diagrams as shown in Figure 3. In the diagrams, the area between the lines corresponds to the stable steady state of the system for some particular choice of parameters; for example, by choosing $K_{1}=10$ and $K_{2}=15$ in Figure 3(a)(i), this corresponds to the absence of infected population stable steady state; and if we increase $K_{1}$ to 20 , the system will experience a transcritical bifurcation and the stable steady state will switch to the presence of infected population.

By comparing Figure 3(a)(i) and (ii), we can see that the absence of the infected population region has been significantly reduced as the line separating the absence and presence of the infected population stable steady state has curved to the left for the amplification agent case. This indicates that it is easier for the infected deer mouse to exist if the non-host species is an amplification agent compared to a dilution agent. This can be seen from the narrower region for the absence of infected population stable steady state in Figure 3(a)(ii) compared to Figure 3(a)(i). Similarly, the line between the absence and presence of infected population stable steady states in Figure 3(b)(ii) was shifted to the left. This resulted in a narrower infectionfree stable region, and a much stronger $q_{1}$ is required for the amplification agent to compensate the growing $K_{1}$ compared to the dilution agent. In Figure 3(c)(ii), the line between the absence and presence of the infected population stable steady states has shifted upwards compared to 
Figure 3(c)(i). This indicates that the dilution agent would only require a lower combination of values of $K_{2}$ and $q_{1}$ to suppress the infection prevalence as opposed to the amplification agent.

In summary, our bifurcation results have shown that a dilution agent is much more efficient in subduing the infection population despite its $q_{1}$ is relatively lower than an amplification agent. Thus, it is important to investigate the relationship between the host and other species when the subject of suppressing infection is of concern.

\section{Discussion}

From the above results, we observed that the role played by the non-host species (as amplification or dilution agent) is equally important as the carrying capacity and the interspecific competition strength of both deer mouse and non-host species in regulating the SNV prevalence. Our results in Figure 2(a) match with Dearing and Dizney [24] findings. They found that climate change, e.g. El Nino, or even a small-scale climate differences [25] is likely to enhance the hantavirus prevalence due to the increase in food availability (carrying capacity) for the host population. Furthermore, our investigations on the interspecific competition strength and the role played by the non-host species in the host population may shed some light onto the observed insignificant change in infection prevalence for site $2-5$ in [14] and the Montana site in [5], despite the presence of high species diversity (indicated by the Simpson index) in those sites. Our results have shown the non-host species as the amplification or dilution agent has dire effects on the host dynamics and a richer biodiversity may not necessarily give rise to a dilution effect but rather the identity of the assemblage species is more crucial in determining such effect [20], e.g. Clay et al. [16] observed that pinyon mice may act as the dilution agent (its population density has a negative relationship with the SNV prevalence) and the kangaroo rats may be the amplification agent (its frequency has positive relationship with SNV prevalence), and Galindo and Krebs [26] observed neither meadow voles nor red-backed voles competitive interactions have any influence on the habitat use and relative abundance of deer mice in the southwestern Yukon.

Our study has a few shortcomings as we only considered non-host species in our theoretical model. Other researchers managed to observe other Peromyscus species [27] or desert woodrat [28], serving as secondary reservoirs to SNV. The presence of secondary host may not necessarily amplify the disease prevalence, and surprisingly, it may serve as a dilution agent, depending on its quality in disease transmission [29]. Perhaps the generalized mathematical model on disease transmission between the population of two host species, proposed in [23], can be of use to describe such phenomenon. Besides that, we did not consider dispersal effect in our model. In an ecological study conducted by Mohd et al. [30], they observed the formation of multi-species communities through the interactions of the bistability phenomenon with the species dispersal mechanisms. In another study, Mohd et al. [31] showed that the inclusion of dispersal effect would reduce competitive exclusion effect between species and promotes multispecies coexistence. These studies highlight the importance of investigating the dispersal effect in a multi-species community. For the case of deer mouse, Falkenberg and Clarke [32] reported a shift in the microhabitat use of deer mice in the presence of Ord's kangaroo rats. Hence, we were unsure how this would affect the SNV prevalence in deer mouse. Furthermore, the different elevations in the environment may contain different amount of carrying capacity. For example, the theoretical spatio-temporal model in [11] observed the existence of "refugia" for 


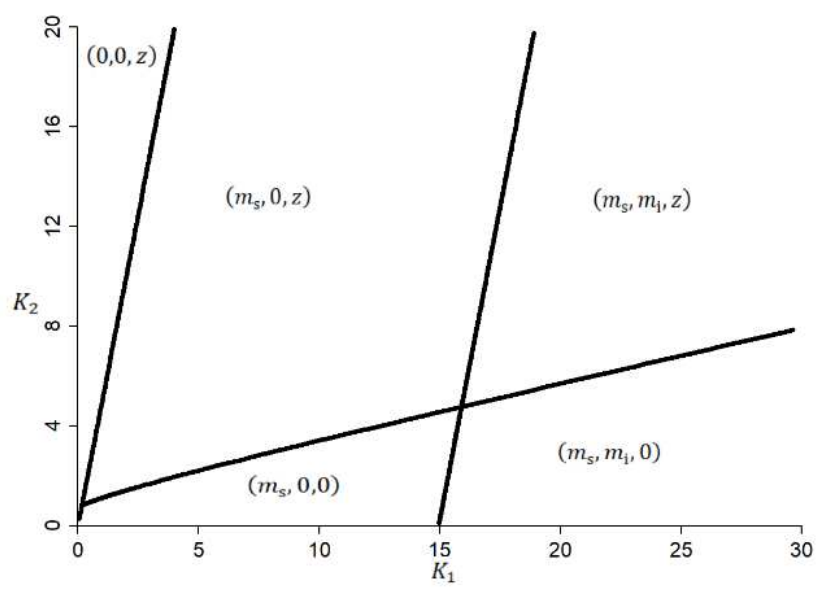

(a)(i)

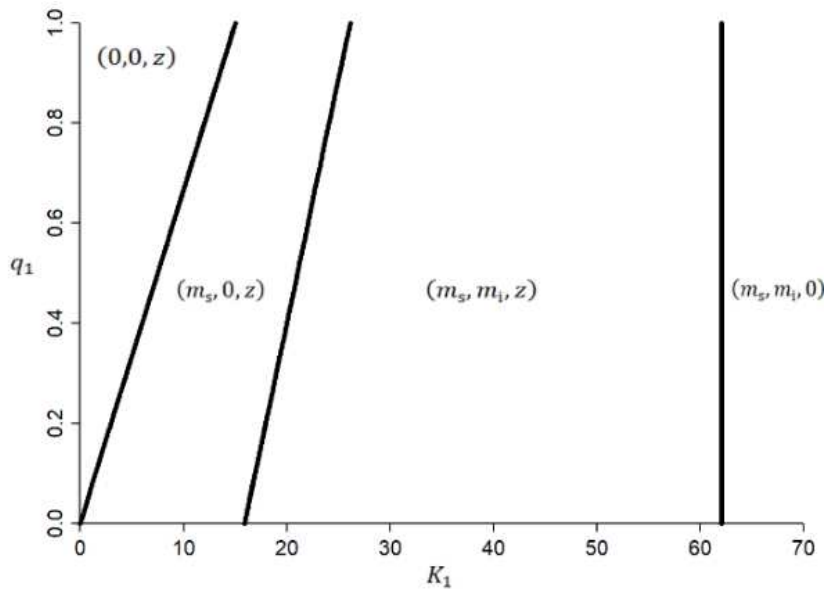

(b)(i)

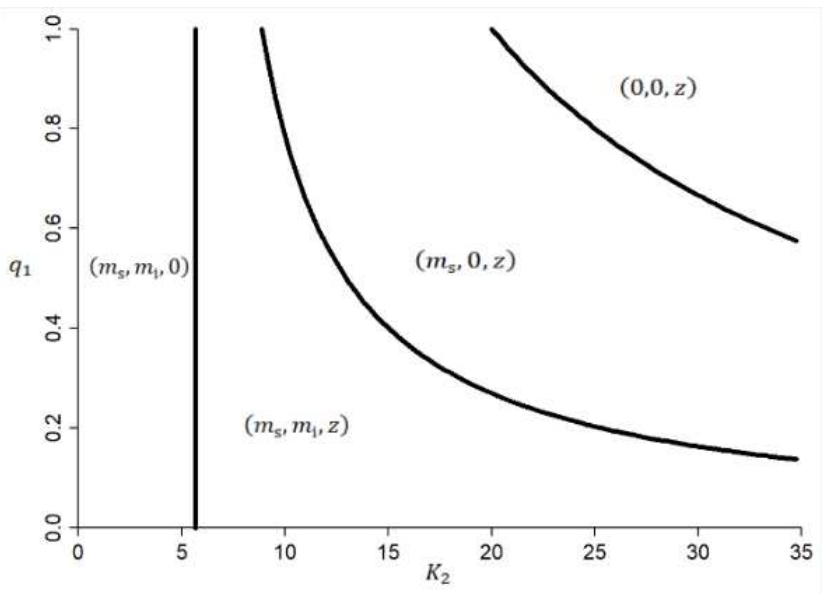

(c)(i)

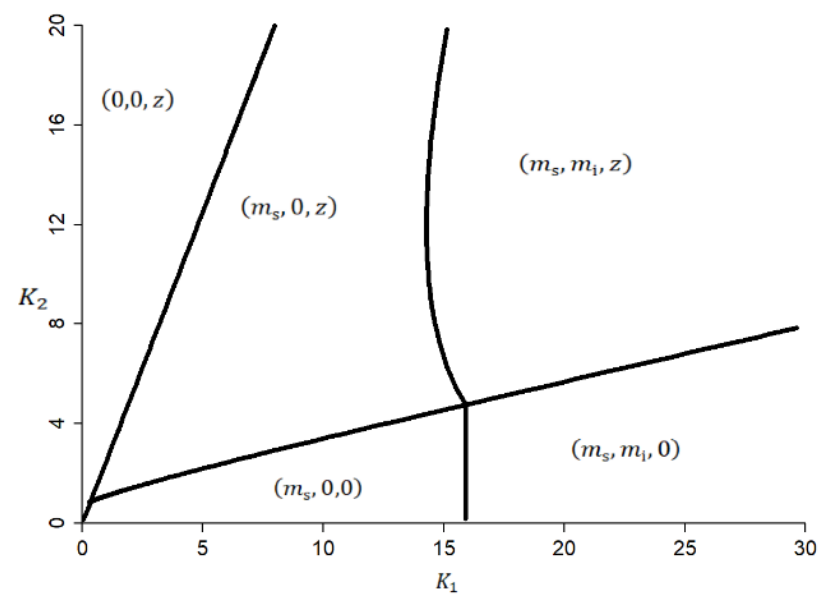

(a)(ii)

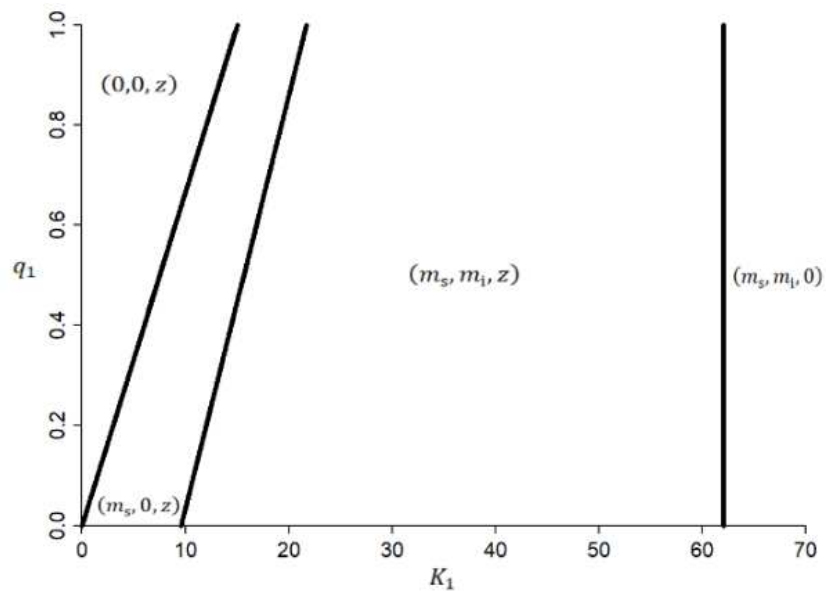

(b)(ii)

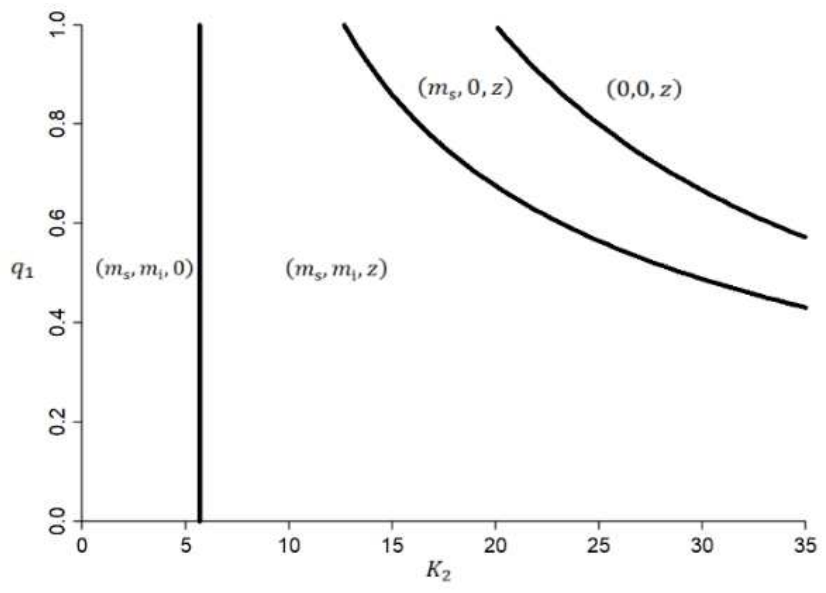

(c)(ii)

Figure 3: Co-dimension 2 bifurcation diagrams where the ones on the left refers to the dilution agent case while the ones on the right refers to the amplification agent case; (a) depicts $K_{1}$ versus $K_{2}$, (b) depicts $K_{1}$ versus $q_{1}$ and (c) depicts $q_{1}$ versus $K_{2}$ 
SNV at the site with higher amount of carrying capacity while the environmental conditions are less favorable at others. Stochastic models can even be considered for further investigations as different findings may be observed between the deterministic and stochastic models. For instance, Mohd et al. [33] observed contrasting results on priority effects in multiple species between their individual based model and deterministic model. Besides that, our study only focused on the competition effect between small mammal species but not on the prey-predator relationship. The effect of prey-predator is of importance as well as a diverse community will surely contain such mechanism and Packer et al. [34] have warned the consequences of predator removal in terms of disease ecology point of view.

\section{Conclusion}

Our study was aimed to highlight the importance of investigating the role played by the nonhost species on the SNV transmission in deer mouse. We analyzed our proposed model in terms of carrying capacity and interspecific competition strength to compare the effect between an amplification agent and a dilution agent. Based on our findings, our proposed theoretical model may explain the lack of reduction in the SNV prevalence despite a relatively high biodiversity observed in some empirical studies. We suggest future empirical studies related to SNV prevalence to investigate the species assemblage, and the mechanisms, which regulate the SNV prevalence in the deer mouse population. With this data, the current mathematical models can then be refined to better reflect the dynamics of the deer mouse population in transmitting SNV.

\section{Acknowledgement}

The authors would like to thank the School of Mathematical Sciences and the Universiti Sains Malaysia (USM) for the support. Mohd Hafiz Mohd is supported by the Universiti Sains Malaysia (USM) Fundamental Research Grant Scheme (FRGS) No. 203/PMATHS/6711645.

\section{References}

[1] Ostfeld, R. S. and Keesing, F. Biodiversity and disease risk: the case of Lyme disease. Conservation Biology. 2000. 14(3): 722-728.

[2] Keesing, F., Holt, R. D. and Ostfeld, R. S. Effects of species diversity on disease risk. Ecology Letters. 2006. 9: 485-498.

[3] Randolph, S. E. and Dobson, A. D. M. Pangloss revisited: a critique of the dilution effect and the biodiversity-buffers-disease paradigm. Parasitology. 2012. 139: 847-863.

[4] Milholland, M. T., Castro-Arellano, I., Arellano, E., Nava-Gracia, E., Rangel-Altamirano, G., Gonzalez-Cozatl, F. X., Suzan, G., Schountz, T., Gonzalez-Padron, S., Vigueras, A., Rubio, A. V., Maikis, T. J., Westrich, B. J., Martinez, III, J. A., Esteve-Gassent, M. D., Torres, M., Rodriguez-Ruiz, E. R., Hahn, D. and Lacher, Jr, T. E. Species identity supersedes the dilution effect concerning hantavirus prevalence at sites across Texas and Mexico. ILAR Journal. 2017. 58(3): 401-412. 
[5] Luis, A. D., Kuenzi, A. J. and Mills, J. N. Species diversity concurrently dilutes and amplifies transmission in a zoonotic host-pathogen system through competing mechanisms. Proceedings of the National Academy of Sciences. 2018. 115(31): 7979-7984.

[6] Peixoto, I. D. and Abramson, G. The effect of biodiversity on the hantavirus epizootic. Ecology. 2006. 87(4): 873-879.

[7] Jiang, H., Zheng, X., Wang, L., Du, H., Wang, P. and Bai, X. Hantavirus infection: a global zoonotic challenge. Virologica. 2017. 32(1): 32-43.

[8] MacNeil, A., Nichol, S. T. and Spiropoulou, C. F. Hantavirus pulmonary syndrome. Virus Research. 2011. 162: 138-147.

[9] Douglass, R. J., Wilson, T., Semmes, W. J., Zanto, S. N., Bond, C. W., Horn, R. C. V. and Mills, J. N. Longitudinal studies of Sin Nombre virus in deer mouse-dominated ecosystems of Montana. The American Journal of Tropical Medicine and Hygiene. 2001. 65(1): 33-41.

[10] Glass, G. E., Yates, T. L., Fine, J. B., Shields, T. M., Kendall, J. B., Hope, A. G., Parmenter, C. A., Peters, C. J., Ksiazek, T. G., Li, C. S., Patz, J. A. and Mills, J. N. Satellite imagery characterizes local animal reservoir populations of Sin Nombre virus in the southwestern United States. Proceedings of the National Academy of Sciences of the United States of America. 2002. 99(26): 16817-16822.

[11] Abramson, G. and Kenkre, V. M. Spatio-temporal patterns in hantavirus infection. Physical Review E. 2002. 66: 011912.

[12] Calisher, C. H., Wagoner, K. D., Amman, B. R., Root, J. J., Douglass, R. J., Kuenzi, A. J., Abbott, K. D., Parmenter, C., Yates, T. L., Ksiazek, T. G. Beaty, B. J. and Mills, J. N. Demographic factors associated with prevalence of antibody to Sin Nombre virus in deer mice in the Western United States. Journal of Wildlife Diseases. 2007. 43(1): 1-11.

[13] Botten, J., Mirowsky, K., Kusewitt, D., Ye, C., Gottlieb, K., Prescott, J. and Hjelle, B. Persistent Sin Nombre virus infection in the deer mouse (Peromyscus maniculatus) model: sites ofreplication and strand-specific expression. Journal of Virology. 2003. 77(2): 1540-1550.

[14] Dizney, L. J. and Ruedas, L. A. Increased host species diversity and decreased prevalence of Sin Nombre Virus. Emergin Infectious Diseases. 2009. 15(7): 1012-1018.

[15] Suzan, G., Marce, E., Giermakowski, J. T., Mills, J. N., Ceballos, G., Ostfeld, R. S., Armien, B., Pascale, J. M. and Yates, T. L. Experimental evidence for reduced rodent diversity causing increased hantavirus prevalence. PLOS ONE. 2009. 4(5): e5461.

[16] Clay, C. A., Lehmer, E. M., Jeor, S. S. and Dearing, M. D. Sin Nombre virus and rodent speciesdiversity: A test the dilution and amplification hypotheses. PLOS ONE. 2009. 4(7): e6467.

[17] Dearing, M. D., Clay, C., Lehmer, E. and Dizney, L. The roles of community diversity and contact rates on pathogen prevalence. Journal of Mammalogy. 2015. 96(1): 29-36.

[18] Gao, L. Q. and Hethcote, H. W. Disease transmission models with density-dependent demographics. Journal of Mathematical Biology. 1992. 30(7): 717-731.

[19] Clay, C. A., Lehmer, E. M., Jeor, S. S. and Dearing, M. D. Testing mechanisms of the dilution effect: Deer mice encounter rates, Sin Nombre virus prevalence and species diversity. EcoHealth. 2009. 6: 250-259. 
[20] Rubio, A. V., Castro-Arellano, I., Mills, J. N., List, R., Avila-Flores, R. and Suzan, G. Is species richness driving intra- and interspecific interactions and temporal activity overlap of a hantavirus host? An experimental test. PLOS ONE. 2017. 12(11): e0188060.

[21] Brenner, G. J. and Moynihan, J. A. Stressor-induced alterations in immune response and viral clearance following infection with Herpes Simplex Virus-Type 1 in BALB/c and C57BI/6Mice. Brain, Behavior, and Immunity. 1997. 11: 9-23.

[22] Luis, A. D., Douglass, R. J., Mills, J. N. and Bjornstad, O. N. Environmental fluctuations lead to predictability in Sin Nombre hantavirus outbreaks. Ecology. 2015. 96(6): 1691-1701.

[23] O'Regan, S. M., Vinson, J. E. and Park, A. W. Interspecific contact and competition may affect the strength and direction of disease-diversity relationships for directly transmittedmicroparasites. The American Naturalist. 2015. 186(4): 480-494.

[24] Dearing, M. D. and Dizney, L. Ecology of hantavirus in a changing world. Annals of the New York Academy of Sciences. 2010. 11951(1): 99-112.

[25] Tersago, K., Schreurs, A., Linard, C., Verhagen, R., Dongen, S. V. and Leirs, H. Population, environmental, and community effects on local bank vole (Myodesglareolus) Puumala virus infection in an area with low human incidence. Vector Borne and Zoonotic Diseases. 2008. 8(2): 235-244.

[26] Galindo, C. and Krebs, C. J. Habitat use and abundance of deer mice: interactions with meadow voles and red-backed voles. Canadian Journal of Zoology. 1984. 63: 1870-1879.

[27] Abbott, K. D., Ksiazek, T. G. and Mills, J. N. Long-term hantavirus persistence in rodent populations in Central America. Emerging Infectious Diseases. 1999. 5(1): 102-112.

[28] Dearing, M. D., Mangione, A. M., Karasov, W. H., Morzunov, S., Otteson, E. and Jeor, S. S. Prevalence of hantavirus in four species of Neotoma from Arizona and Utah. Journal of Mammalogy. 1998. 79(4): 1254-1259.

[29] Ostfeld, R. S. and Keesing, F. Effects of host diversity on infectious disease. Annual Reviewof Ecology, Evolution, and Systematics. 2012. 43: 157-182.

[30] Mohd, M. H., Murray, R., Plank, M. J. and Godsoe, W. Effects of different dispersal patterns on the presence-absence of multiple species. Commun. Nonlinear Sci. Numer. Simulat. 2018. 56: 115-130.

[31] Mohd, M. H., Murray, R., Plank, M. J. and Godsoe, W. Effects of biotic interactions and dispersal on the presence-absence of multiple species. Chaos, Solitons \& Fractals. 2017. 99: 185-194.

[32] Falkenberg, J. C. and Clarke, J. A. Microhabitat use of deer mice: effects of interspecificinteraction risks. Journal of Mammalogy. 1998. 79(2): 558-565.

[33] Mohd, M. H., Murray, R., Plank, M. J. and Godsoe, W. Effects of dispersal and stochasticity onthe presence-absence of multiple species. Ecological Modelling. 2016. 342: 49-59.

[34] Packer, C., Holt, R. D., Hudson, P. J., Lafferty, K. D. and Dobson, A. P. Keeping the herds healthy and alert: implications of predator control for infectious disease. Ecology Letters. 2003. 6: 797-802. 